hep-th/0302200

HU-EP-03-11

\title{
BOUNDARIES, DEFECTS AND FROBENIUS ALGEBRAS
}

\author{
Jürgen Fuchs ${ }^{1}$ Ingo Runkel ${ }^{2,3}$ and Christoph Schweigert ${ }^{2,4}$ \\ ${ }^{1}$ Institutionen för fysik, Universitetsgatan 5, S-651 88 Karlstad \\ ${ }^{2}$ LPTHE, Université Paris VI, 4 place Jussieu, F-75 252 Paris Cedex 05 \\ ${ }^{3}$ Institut für Physik, HU Berlin, Invalidenstraße 110, D-10115 Berlin \\ ${ }^{4}$ Institut für theoretische Physik E, RWTH Aachen, Sommerfeldstr. 28, D-52074 Aachen
}

\begin{abstract}
The interpretation of D-branes in terms of open strings has lead to much interest in boundary conditions of two-dimensional conformal field theories (CFTs). These studies have deepened our understanding of CFT and allowed us to develop new computational tools. The construction of CFT correlators based on combining tools from topological field theory and non-commutative algebra in tensor categories, which we summarize in this contribution, allows e.g. to discuss, apart from boundary conditions, also defect lines and disorder fields.
\end{abstract}




\section{Basic CFT facts}

In the applications of two-dimensional conformal field theory - two-dimensional critical systems, quasi one-dimensional condensed matter physics, and string theory - boundary effects play an important role. They appear in the description of pointlike defects such as the Kondo effect, the computation of percolation probabilities, the construction of type I superstring theories, and the formulation of open string perturbation theory in the background of D-branes. The study of boundary conditions has very much improved our understanding of the structure of CFT; this is one topic we address in the present contribution. But two-dimensional conformal field theories possess an extremely rich structure, which is in fact not exhausted by bulk and boundary fields. In particular, they also involve defect lines and disorder fields; these objects will be discussed as well. Hitherto, they have not yet played a role in world sheet theories of strings.

Let us briefly review a few pertinent aspects of CFT. In the bulk there are both left moving and right moving degrees of freedom, which are described by a left and a right moving chiral algebra, respectively. Each of them contains the Virasoro algebra, which realizes the conformal symmetry, given by generators $\left\{L_{n} \mid n \in \mathbb{Z}\right\}$ with relations

$$
\left[L_{n}, L_{m}\right]=(n-m) L_{n+m}+\frac{c}{12} n\left(n^{2}-1\right) \delta_{n,-m}
$$

It is natural to study the representations of the chiral algebra; they provide the superselection sectors of the chiral CFT. Primary fields correspond to irreducible representations; if their number is finite, the theory is called rational. With the help of the representation theory one can introduce conformal blocks which are spaces of multivalued functions of insertion points on a complex curve. For WZW theories, they can be computed as (subspaces of) the spaces of solutions to Knizhnik-Zamolodchikov equations. For the full local conformal field theory they play the role of precorrelators; they also form the state spaces of the associated three-dimensional topological field theory. The monodromy properties of these conformal blocks provide us with a collection of data - fusing matrices, braiding matrices, conformal weights, representations of mapping class groups - that encode the basic information about the chiral CFT; they are usually referred to as chiral data, or also as Moore-Seiberg data.

A good handle on these data is an essential prerequisite for any powerful approach to CFT. Technically, it is afforded by the so-called modular tensor categories. They furnish a basisindependent formalization of the chiral data, which are obtained from solutions to differential equations, in much the same way as the notion of a vector space constitutes a basis-independent description for the structure of the space of solutions to linear equations.

The representations of a chiral algebra form a category; its objects are representations, its morphisms are intertwining maps between representations. Here the term 'chiral algebra' should be taken with some care - we do not necessarily start with a maximally extended chiral algebra, for left movers or for right movers. This gives us sufficient flexibility to address symmetry breaking boundary conditions as well as situations in which left moving and right moving chiral algebra do not coincide.

The representation category of a chiral algebra comes with a lot of structure:

- The notion of a tensor product, or fusion, of representations leads to a tensor product

$$
\otimes: \quad \mathcal{C} \times \mathcal{C} \rightarrow \mathcal{C}
$$


for the category. For this tensor product, the vacuum sector acts as a unit.

- Braid group statistics in low dimensions provides us with the notion of a braiding, which constitutes a systematic prescription to permute representations in tensor products that is compatible with the braid group relations.

- The concept of dual representations gives rise to a pairing of representations.

These structures obey a number of compatibility relations, and these are summarized in the definition of a modular tensor category. The qualification 'modular' indicates the fact that from such a category one can infer a natural candidate for the action of the modular group, i.e. the mapping class group of the torus, on the characters of the CFT. Modular tensor categories also arise as (truncations of) categories of representations of quantum groups, and as categories of representations of weak Hopf algebras. It is an important result [16] that to every modular tensor category one can associate a topological field theory in three dimensions. This realizes a "holographic" correspondence between chiral CFT in two dimensions and TFT in three dimensions.

\section{Algebra and topological field theory}

The central idea of our approach to (rational) conformal field theory is to combine tools from topological field theory with non-commutative algebra in tensor categories.

The central insight can be summarized as follows:

For a given chiral algebra, a full local CFT can be constructed from the algebra of open string states for a single boundary condition ( = D-brane).

To understand this result, let us examine the algebra of open string states, i.e. the algebra of boundary operators $\Psi_{a}^{A A}$ that respect a given boundary condition $A$, in more detail:

- The field-state correspondence allows us to work with the corresponding states. They form a module over the chiral algebra, i.e. a (generically reducible) object in the modular tensor category $\mathcal{C}$; we use the symbol $A$ also to denote this object.

- The operator product

$$
\Psi_{a}^{A A}(x) \Psi_{b}^{A A}(y) \sim \sum_{c} \mathrm{C}_{a b}^{c}(x-y)^{\Delta_{c}-\Delta_{a}-\Delta_{b}} \Psi_{c}^{A A}(y)
$$

of boundary fields is associative. This leads to an equation of the type

$$
\mathrm{CC}=\sum \mathrm{CCF} \text {. }
$$

It is a first crucial observation that this relation gives rise to an associative multiplication morphism $m \in \operatorname{Hom}(A \otimes A, A)$ on the object $A$. The presence of the fusing matrix $F$ in the relation for the structure constants $C$ is taken into account by a non-trivial notion of associativity in the category $\mathcal{C}$.

- Another important aspect of the algebra object $A$ is the fact that the non-degeneracy of the two-point functions of boundary fields on the disk implies that $A$ can be endowed with the structure of a Frobenius algebra, i.e. an algebra with a non-degenerate invariant bilinear form. (For precise definitions, see [9]). Actually, $A$ is even a so-called symmetric special Frobenius algebra. It should be appreciated, though, that $A$ is not necessarily (braided-) commutative, as 
befits an algebra of boundary fields. Moreover, the boundary condition we start with need not be elementary; it may equally well be a superposition of several elementary boundary conditions, including possibly Chan-Paton multiplicities.

- Finally, for any other boundary condition $M$ the associativity of the operator product

$$
\Psi^{A A} \Psi^{A A} \Psi^{A M}
$$

of boundary fields allows us to abstract a map $\rho \in \operatorname{Hom}(A \otimes M, M)$ that endows the corresponding object $M$ of $\mathcal{C}$ with the structure of a (left) $A$-module. We thus learn that boundary conditions are given by $A$-modules.

Let us now discuss the second input of our construction, topological field theory. As a direct consequence of the axioms, every modular tensor category allows for the construction of a three-dimensional TFT. The latter assigns to every closed oriented two-manifold $\hat{\Sigma}$ a finite-dimensional vector space $\mathcal{H}(\hat{\Sigma})$, the space of conformal blocks, on which the mapping class group $\operatorname{Map}(\hat{\Sigma})$ acts projectively. Further, to every three-manifold with boundary $\hat{\Sigma}$ that contains a Wilson graph, the TFT assigns a vector in $\mathcal{H}(\hat{\Sigma})$. The axioms of a TFT formalize the well-known relation between a TFT on a three-dimensional manifold and a chiral CFT on its boundary, a structure that is e.g. central to the CFT description of universality classes of quantum Hall fluids (for a review, see e.g. [7]).

\section{The TFT construction of CFT correlators}

Our goal is now to determine CFT correlators on a surface $\Sigma$ that possibly has a boundary. To be able to apply tools from TFT, we need to work with a suitable two-manifold $\hat{\Sigma}$ without boundary. A natural candidate for $\hat{\Sigma}$ is the complex double [3] of $\Sigma$. It comes with an orientation reversing involution $\sigma$ such that $\Sigma$ is identified with the quotient of $\hat{\Sigma}$ by the action of $\sigma$. Indeed, it is natural [15] to view the world sheet $\Sigma$ as a real scheme and $\hat{\Sigma}$ as its complexification; the orientifold map $\sigma$ is then just the action of the non-trivial element of the Galois group $\operatorname{Gal}(\mathbb{C} / \mathbb{R}) \cong \mathbb{Z}_{2}$.

With the help of the double cover $\hat{\Sigma}$, we can in particular give a concise version of the principle of holomorphic factorization: The correlators of the CFT on $\Sigma$ are specific vectors in the space $\mathcal{H}(\hat{\Sigma})$ of conformal blocks on the complex double, which are determined by two types of constraints:

(1) They must be invariant under the action of $\operatorname{Map}(\Sigma) \cong \operatorname{Map}(\hat{\Sigma})^{\sigma}$.

(2) They must satisfy factorization rules.

According to the principle of holomorphic factorization we need to select a specific element of $\mathcal{H}(\hat{\Sigma})$ to describe a correlator on $\Sigma$. By the principles of TFT, in turn, such a vector is determined by a three-manifold $\mathrm{M}_{\Sigma}$ whose boundary is the double $\hat{\Sigma}$ and a ribbon graph in $\mathrm{M}_{\Sigma}$. For $\mathrm{M}_{\Sigma}$ we take the so-called connecting manifold [6], defined as the quotient of the interval bundle $\hat{\Sigma} \times[-1,1]$ by the $\mathbb{Z}_{2}$ that acts as $\sigma$ on $\hat{\Sigma}$ and as $t \mapsto-t$ on the interval. The points with $t=0$ provide a distinguished embedding of $\Sigma$ into $\mathrm{M}_{\Sigma}$. In fact, $\Sigma$ is a retract of $\mathrm{M}_{\Sigma}$ or, in more intuitive terms, $M_{\Sigma}$ is just a fattening of the world sheet $\Sigma$. (To give one example: the double of a disk is a sphere, and the orientifold map is the reflection at the equatorial plane; the connecting manifold is in this case a full three-ball.) Concerning the prescription for the ribbon graph in $\mathrm{M}_{\Sigma}$ we refer to [8]. We merely recall that it involves a (dual) triangulation of 
$\Sigma$ with the ribbons labelled by the Frobenius algebra object $A$ and with the trivalent vertices corresponding to the multiplication morphism $m$ of $A$.

In this framework, the consistency relations of modular invariance (1) and factorization (2) can be proven rigorously. We also recover the various combinatorial data - such as modular invariant partition functions, NIM-reps and classifying algebras - that arise when studying specific small subsets of these consistency conditions (see e.g. [13, 10, 2]). Moreover, Morita equivalence, combined with orbifold technology, allows for an elegant proof of T-dualities for arbitrary topology of the world sheet.

We have already seen that modules over $A$ correspond to boundary conditions. In noncommutative algebra, it is natural to consider bimodules as well, and again they turn out to possess a physical interpretation: they describe defect lines. More specifically, these defect lines carry neither momentum nor charges. Thus they are topological in nature, so that their position matters only up to homotopy. These defect lines can actually be generalized to interfaces between two different full local CFTs that are based on one and the same chiral CFT. Disorder fields create or change defect lines.

The following table, associating algebraic structures to physical concepts, can serve as a succinct summary of our results [9]:

\begin{tabular}{l|l}
\multicolumn{1}{c|}{ Physical concept } & \multicolumn{1}{|c}{ Algebraic structure } \\
\hline Boundary condition & left $A$-module \\
Boundary field $\Psi_{a}^{M N}$ & $\operatorname{Hom}_{A}(M \otimes a, N)$ \\
\hline Bulk field $\Phi_{a b}$ & $\operatorname{Hom}_{A, A}\left((A \otimes a)^{-},(A \otimes b)^{+}\right)$ \\
Defect line & $A$-bimodule \\
Disorder field $\Phi_{a b}^{B_{1} B_{2}}$ & $\operatorname{Hom}_{A, A}\left(\left(B_{1} \otimes a\right)^{-},\left(B_{2} \otimes b\right)^{+}\right)$
\end{tabular}

In particular, bulk fields can be regarded as a special type of disorder fields - those which connect the trivial defect line, i.e. the one labelled by $A$, to the trivial defect line.

\section{An example: Permutation branes}

Our algebraization of physical concepts not only leads to statements that can be proved rigorously, but also allows us to establish powerful algorithms for doing computations. In particular, for constructing a full local CFT only a single non-linear constraint needs to be solved: the one that encodes associativity of the Frobenius algebra object $A$. We illustrate the power of our approach with the following example.

Consider the tensor product of $N$ identical conformal field theories whose modular tensor category is $\mathcal{C}$. We are then dealing with the category $\mathcal{C}^{\times N}$, which comes with an action of the symmetric group $S_{N}$. One may wish to classify all those boundary conditions of the $\mathcal{C}^{\times N_{-}}$ theory which respect the subalgebra of the chiral algebra that is left pointwise fixed under the $S_{N}$-action. To perform this task we can work with the orbifold category

$$
\mathcal{C}_{\text {orb }}:=\mathcal{C}^{\times N} / S_{N}
$$

for which many aspects of the chiral data are explicitly known 4, 1. In particular, the primary fields of the orbifold theory are labelled by pairs $(\{\vec{\lambda}\}, \psi)$, where $\{\vec{\lambda}\}$ is an unordered $N$-tuple 
of labels for simple objects in $\mathcal{C}$ and $\psi$ is a character of the double of the stabilizer subgroup $S_{\vec{\lambda}}$ of $\{\vec{\lambda}\}$ in $S_{N}$.

It is not difficult to find the relevant algebra object. The algebra structure is determined by the product in the algebra of functions on $S_{N}$, and it is realized on the object

$$
A=\bigoplus_{\psi \in S_{N}^{*}} \operatorname{dim} V_{\psi}(\{\overrightarrow{0}\},(\psi,[e])) .
$$

Here $\overrightarrow{0}$ is the $N$-tuple each entry of which is the vacuum sector of $\mathcal{C}$. Its stabilizer is all of $S_{N}$; the pairs $(\psi,[e])$, consisting of a character $\psi$ of $S_{N}$ and the conjugacy class of the unit element $e \in S_{N}$, specify a subclass of irreducible representations of the double $\mathcal{D}\left(S_{N}\right)$.

One can show that $A$ is a (braided-) commutative symmetric special Frobenius algebra. The irreducible $A$-modules, i.e. the "permutation branes" in the terminology of [14], can be easily determined with the help of theorem 3.2 of [12]. They are labelled by pairs $(\vec{\lambda}, g)$ where $\vec{\lambda}$ is now an ordered $N$-tuple of labels and $g$ is an element of the stabilizer group $\mathcal{S}_{\vec{\lambda}}$. One can then apply the formalism of [8, 9] to compute e.g. the boundary states and annulus partition functions. The latter turn out to be linear combinations of twining characters with prefactors that are sums of products of fusion rules of the $\mathcal{C}$-theory. The NIM-rep property of the annuli as well as the consistency of the boundary states is guaranteed by the general theory.

\section{Conclusions}

Besides its computational power and the possibility to obtain general proofs of consistency, an additional benefit of our approach is that it reduces old physical questions to standard problems in algebra and representation theory. Here are some interesting examples:

- The classification of CFTs with given chiral data $\mathcal{C}$ amounts to classifying Morita classes of symmetric special Frobenius algebras in the category $\mathcal{C}$. In particular, physical modular invariants of automorphism type are classified by the Brauer group of $\mathcal{C}$.

- The classification of boundary conditions and defect lines is reduced to the standard representation theoretic problem of classifying modules and bi-modules. As a consequence, powerful methods like induced modules and reciprocity theorems are at our disposal.

- The problem of deforming CFTs is related to the problem of deforming algebras, which is a cohomological question. For the moment, the only known results in this direction are rigidity theorems [5]: a rational CFT cannot be deformed within the class of rational CFTs. It will therefore be important to get a better understanding of non-rational CFT. 


\section{References}

[1] P. Bantay, Characters and modular properties of permutation orbifolds, Phys. Lett. B 419 (1998) 175

[2] R.E. Behrend, P.A. Pearce, V.B. Petkova, and J.-B. Zuber, Boundary conditions in rational conformal field theories, Nucl. Phys. B 579 (2000) 707

[3] S.K. Blau, M. Clements, S. Della Pietra, S. Carlip, and V. Della Pietra, The string amplitude on surfaces with boundaries and crosscaps, Nucl. Phys. B 301 (1988) 285

[4] L.A. Borisov, M.B. Halpern, and C. Schweigert, Systematic approach to cyclic orbifolds, Int. J. Mod. Phys. A 13 (1998) 125

[5] P. Etingof, D. Nikshych, and V. Ostrik, On fusion categories, preprint math.QA/0203060

[6] G. Felder, J. Fröhlich, J. Fuchs, and C. Schweigert, Conformal boundary conditions and three-dimensional topological field theory, Phys. Rev. Lett. 84 (2000) 1659;

Correlation functions and boundary conditions in RCFT and three-dimensional topology, Compos. Math. 131 (2002) 189

[7] J. Fröhlich, B. Pedrini, C. Schweigert, and J. Walcher, Universality in quantum Hall systems: coset construction of incompressible states, J. Stat. Phys. 103 (2001) 527

[8] J. Fuchs, I. Runkel, and C. Schweigert, Conformal correlation functions, Frobenius algebras and triangulations, Nucl. Phys. B 624 (2002) 452

[9] J. Fuchs, I. Runkel, and C. Schweigert, TFT construction of RCFT correlators I: Partition functions, Nucl. Phys. B 646 (2002) 353

[10] J. Fuchs and C. Schweigert, A classifying algebra for boundary conditions, Phys. Lett. B 414 (1997) 251

[11] J. Fuchs and C. Schweigert, Lie algebra automorphisms in conformal field theory, Contemp. Math. 297 (2002) 117

[12] A.A. Kirillov, Modular categories and orbifold models, preprint math.QA/0104242

[13] G. Pradisi, A. Sagnotti, and Ya.S. Stanev, Completeness conditions for boundary operators in 2D conformal field theory, Phys. Lett. B 381 (1996) 97

[14] A. Recknagel, Permutation branes, preprint hep-th/0208119

[15] C. Schweigert and J. Fuchs, The world sheet revisited, preprint hep-th/0105266, to appear in Fields Institute Commun.

[16] V.G. Turaev, Quantum Invariants of Knots and 3-Manifolds (de Gruyter, New York 1994) 